Bulletin of Mathematical Biology (2000) 62, 501-525

doi:10.1006/bulm.1999.0166

Available online at http://www.idealibrary.com on IDE $\mathbf{A}$.

\title{
A Chemotactic Model for the Advance and Retreat of the Primitive Streak in Avian Development
}

\author{
K. J. PAINTER AND P. K. MAINI ${ }^{\dagger}$ \\ Centre for Mathematical Biology, \\ Mathematical Institute, \\ 24-29 St Giles', \\ Oxford, OX1 3LB, \\ U.K \\ H. G. OTHMER \\ Department of Mathematics, \\ University of Minnesota, \\ Minneapolis, MN, 55407, \\ U.S.A.
}

The formation of the primitive streak in early avian development marks the onset of gastrulation, during which large scale cell movement leads to a trilaminar blastoderm comprising prospective endodermal, mesodermal and ectodermal tissue. During streak formation a specialized group of cells first moves anteriorly as a coherent column, beginning from the posterior end of the prospective anteriorposterior axis (a process called progression), and then reverses course and returns to the most posterior point on the axis (a process called regression). To date little is known concerning the mechanisms controlling either progression or regression. Here we develop a model in which chemotaxis directs the cell movement and which is capable of reproducing the principal features connected with progression and regression of the primitive streak. We show that this model exhibits a number of experimentally-observed features of normal and abnormal streak development, and we propose a number of experimental tests which may serve to illuminate the mechanisms. This paper represents the first attempt to model the global features of primitive streak formation, and provides an initial stage in the development of a more biologically-realistic discrete cell model that will allow for variation of properties between cells and control over movement of individual cells.

(C) 2000 Society for Mathematical Biology

\section{INTRODUCTION}

The chick embryo develops from a small, disk-shaped blastodisc floating on top of the yolk. After fertilization cells divide repeatedly, forming a multicellular stratified structure called the blastoderm. The period from just prior to laying

\footnotetext{
${ }^{\dagger}$ Author to whom correspondence should be addressed. Current address: Department of Mathematics, University of Minnesota, Minneapolis, MN.

$0092-8240 / 00 / 030501+25 \quad \$ 35.00 / 0$

(C) 2000 Society for Mathematical Biology
} 
through several hours afterwards has been subdivided into 14 stages (Eyal-Giladi and Kochav, 1976; Khaner, 1993). Cell division is dominant during stages I-VI, and morphogenetic movements begin during stages VII-X, when cells of the central blastodisc, called the area pellucida, separate from the yolk, producing a hollow region beneath the disc called the subgerminal cavity (Penner and Brick, 1984; Stern, 1990). Subsequently, some cells from the central blastodisc move into the subgerminal cavity (either actively or passively), and simultaneously the disc expands radially over the yolk. The opaque marginal zone of the blastoderm, known as the area opaca, remains in contact with the yolk and may play an active role in the radial movement. The result is that during stages VII-X the central part of the disc changes from a layer $4-6$ cells deep to a translucent layer one cell thick called the epiblast. The anterior-posterior axis of the embryo is also determined during these stages (Khaner, 1993). After stage X some cells within the marginal zone migrate posteriorly, and then leave the marginal zone at the posterior marginal zone (PMZ). They spread across the subgerminal cavity beneath the epiblast as a loosely-connected sheet, incorporating islands of cells shed from the blastodisc earlier. By stage XIV this sheet connects with the anterior margin of the disc and forms the hypoblast, and at this stage the blastoderm is bi-layered with the epiblast and hypoblast separated by the blastocoel cavity. Fate maps for cell movements in these stages are available (Hatada and Stern, 1994).

During hypoblast formation the embryonic shield or Koller's sickle develops at the posterior end of the epiblast. This consists of a thickened epiblast (Spratt, 1942) comprising primitive streak precursor cells that have migrated to this area by a series of 'polonnaise movements' (Vakaet, 1984). The first visible sign of gastrulation is formation of the primitive streak, which arises from Koller's sickle at the posterior midline of the blastodisc (Khaner, 1998). The sickle narrows and the primitive streak moves anteriorly between the epiblast and the hypoblast. The tip of the ingressing streak, called Hensen's node, moves $\sim 60 \%$ of the way across the blastoderm before it stops, and later, regresses. The appearance of the blastoderm at the maximal forward extension of the streak is shown in Fig. 1. During advance of the node epiblast cells move into the streak, and those that migrate through the node form anterior structures, those that migrate through the lateral parts of the primitive streak become endodermal and mesodermal cells, and the remainder constitute the ectoderm. Simultaneously, the area pellucida changes from circular to pear shaped, narrowing in the posterior portion. The head structure, notochord and somites are laid down during regression of the node, and when regression is complete the embryo is a flat trilaminar blastoderm comprising the ectodermal, mesodermal and endodermal layers. These will form various organs during subsequent morphogenesis, in addition to the structures formed during regression. The regressing node and anterior portion of the streak eventually form the tail bud (Spratt, 1947). Regression takes place on a slower time scale than progression, taking some 20 hours to regress from its maximum length of $1.9 \mathrm{~mm}$ (Spratt, 1947). 


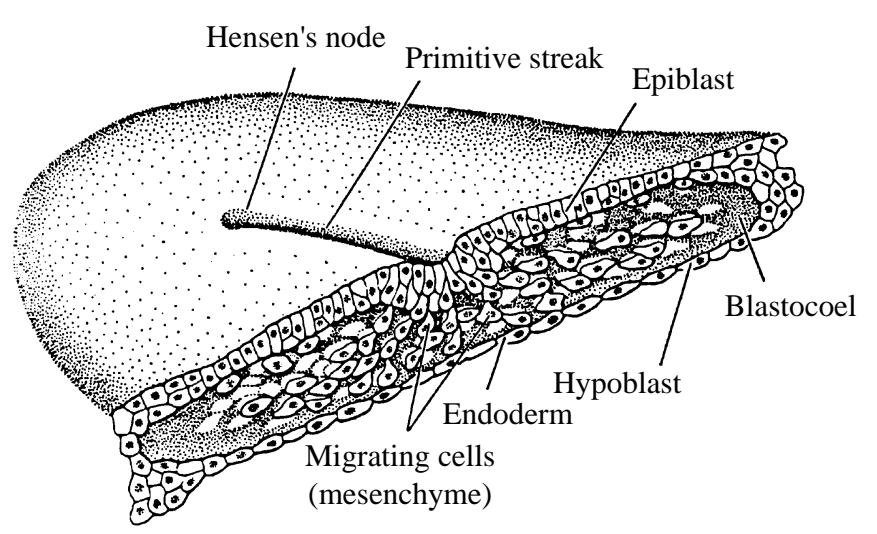

Figure 1. A schematic of the blastoderm at the time of maximal extension of the streak. As described in the text, epiblast cells move into the blastocoel through the primitive streak. From (Gilbert, 1991), with permission.

Although the sequence of events in early avian development is well documented, less is known about the mechanisms that give rise to primitive streak formation and movement, and to date there are no models of these processes. We first focus on the formation and ingression of the primitive streak, as these processes are amenable to experimental manipulation. It is known that Koller's sickle begins to form at the posterior marginal zone at stage $\mathrm{X}$, and if cell movement in this area is blocked, no primitive streak is formed (Spratt, 1966). The ability to promote primitive streak formation depends on position within the marginal zone and on the stage as follows (Khaner and Eyal-Giladi, 1986).

- At stage X only cells in the posterior marginal zone can form the primitive streak; at stage XI the inner region in contact with the posterior marginal zone also has the potential to form a primitive streak, and at stage XII the posterior marginal zone has lost the ability to induce a primitive streak.

- If a fragment of the posterior marginal zone is removed and replaced by lateral marginal zone tissue at stage $\mathrm{X}$, a single primitive streak always originates in the normal position, but if the fragment of posterior marginal zone is replaced by beads which prevent healing of the wound, two primitive streaks form (Khaner and Eyal-Giladi, 1989).

- If donor posterior marginal zone tissue is inserted at $90^{\circ}$ to the host posterior marginal zone at stage $\mathrm{X}$, a single primitive streak develops at the site of the host posterior marginal zone. However, if the host posterior marginal zone is removed two small primitive streaks develop, one at the normal site and one at the transplant site.

These experiments suggest that areas of the marginal zone lateral to the primitive streak can form a primitive streak if they are exposed to fragments of posterior marginal zone, but they are inhibited from doing so by neighboring posterior 
marginal zone. Thus cells in the posterior marginal zone are already differentiated from those in the marginal zone and the remainder of the blastoderm when ingression of the primitive streak begins. The experiments also raise the following questions.

- What initiates motion and guides the early migration of cells in the marginal zone toward the posterior marginal zone?

- What cues guide elongation and movement of the primitive streak? A simple anterior-posterior gradient of a diffusible morphogen cannot be used for positional information along that axis (Khaner and Eyal-Giladi, 1986), for if it were the $90^{\circ}$ transplants of the primitive streak would ingress toward the anterior pole rather than along a ray through the center of the disk.

- What mechanisms can account for the fact that the primitive streak maintains its rod-like structure during ingression? Does the primitive streak ingress by convergent extension (Keller et al., 1991), whereby cells intercalate at the posterior marginal zone and push the primitive streak forward? Are there adhesive differences between cells in the primitive streak and those in the hypoblast and epiblast, or is the structure maintained by chemotactic attraction between cells in the primitive streak?

Traditionally, the blastoderm has been considered homogeneous prior to streak formation, but recent findings suggests earlier cell diversity and significant cell movement near the center of the disc (Shah et al., 1997). Canning and Stern (1988) identified a subpopulation of cells testing positive for the epitope HNK-1, which is first expressed on the surface of cells of the posterior marginal zone and on those which later form the primary hypoblast. Later it is found in the area of streak formation, distributed with a distinct anterior-posterior gradient. A primitive streak does not form when these cells are removed. This has led to the suggestion that HNK-1 cells are the source of streak-derived tissue (Stern and Canning, 1990). The precise role of the epitope itself is not clear, but it may have a role in modulating adhesion [see Stern (1991) and references therein].

Stimulated in part by the wealth of data unearthed in other model developmental systems, many recent experiments have been directed at discovering the genes regulating development. A number of genes have a potential role in development, including members of the homeobox family (Lemaire and Kessel, 1997), the wnt family (Hume and Dodd, 1993), and the caudal genes (Marom et al., 1997). For example, the homeobox gene goosecoid is first found in a small population of cells corresponding to Koller's sickle, (Izpisua-Belmonte et al., 1993). Later this gene characterizes cells of the primitive streak, and expression is highest in cells of Hensen's node and the anterior portion of the streak. Labeling cells of Koller's sickle prior to streak formation reveals that these are the cells subsequently found in anterior portions of the streak, indicating that cells of Koller's sickle have the ability to initiate a primitive streak. This hypothesis is supported by the development 
of an ectopic primitive streak when grafted to lateral regions of a host blastoderm (Izpisua-Belmonte et al., 1993; Callebaut and Nueten, 1994).

The signals involved in streak formation, particularly the transforming growth factors, have also been studied recently. Activin has been shown to induce development of axial structures (Mitrani and Shimoni, 1990; Ziv et al., 1992; Cooke, 1998a), but it does not have the spatial and temporal distribution expected of an inducer. Another member of the $T G F \beta$ family, $c V g l$, expressed in the posterior marginal zone of pre-primitive streak embryos has been shown to induce development of an ectopic primitive streak (Shah et al., 1997).

In summary, development of the streak involves intriguing spatio-temporal patterning properties, and many aspects are as yet poorly understood. Our purpose here is to develop a model for streak movement that can account for some of the principal experimental observations, and to suggest several experiments that can be done to test the model. We do not claim to 'explain' streak ingression and regression, but rather we suggest possible mechanisms in the hope of stimulating experiments aimed at identifying the mechanism actually employed.

\section{DeVelopment of THE MOdel}

Chemotaxis is a widely-used mechanism for directed cell movement and has been extensively studied from both the experimental and theoretical perspectives (Armitage and Lackie, 1990; Alt et al., 1997). In some systems cells merely respond to the external signal without altering it, but in others, such as Dictyostelium discoideum, cells produce and destroy the signal, thereby altering the extracellular signal and affecting cell movement either up (chemoattraction) or down (chemorepulsion) the gradient. While it is not known that chemotaxis is involved in primitive streak formation, it provides a plausible mechanism for producing directed cell movement, and we incorporate it into the model developed here as a means of producing forward and reverse wave movement.

The model is described by the density $n(\mathbf{x}, t)$ of a subpopulation of cells that comprise the primitive streak and which respond to a chemoattractant, whose density is denoted $u(\mathbf{x}, t)$. The assumption that such a subpopulation of cells exists is reasonable in light of the evidence described earlier for variations in cell density and the ability to initiate streak formation ectopically. The identification of cell markers, for example the epitope HNK-1, or the spatial distribution of cells expressing specific genes, for example $g s c$, also suggests that such a population may exist.

The model is described by evolution equations for the cell density $n(\mathbf{x}, t)$ and the density of the attractant $u(\mathbf{x}, t)$. We allow for cell growth and division in the equation for the former, and for degradation of the attractant by cells in the equation for the latter. We denote the underlying domain $\Omega$, the growth rate of the cells by $f_{1}(n, u)$ and the net production of the attractant by $f_{2}(n, u)$. Later we describe 
how the attractant could be produced by all cells in the epiblast. The equations that describe the model are

$$
\begin{aligned}
& \frac{\partial n}{\partial t}=\nabla \cdot\left[D_{n} \nabla n-n \chi(u) \nabla u\right]+f_{1}(n, u), \\
& \frac{\partial u}{\partial t}=D_{u} \nabla^{2} u+f_{2}(n, u),
\end{aligned}
$$

where $D_{u}$ and $D_{n}$ are constant diffusion coefficients and $\chi(u)$ represents the chemotactic sensitivity. A chemotatic response as incorporated in the first equation was first derived by Patlak (1953), by considering a biased random walk model of cell movement, and since then has been used in a similar form by many others (Keller and Segel, 1970; Alt, 1980; Othmer and Stevens, 1997). For simplicity we hereafter assume that the sensitivity $\chi_{0}$ is constant; more general models are described in (Othmer and Stevens, 1997).

\section{Progression and Regression in One Space Dimension}

To develop a qualitative understanding of the model behavior, we first consider a one-dimensional domain $[0, L]$, which represents the posterior-anterior axis of the area pellucida. We use initial conditions

$$
\begin{aligned}
& n(x, 0)= \begin{cases}n_{i} & \text { if } x<x_{i} \\
0 & \text { if } x \geq x_{i},\end{cases} \\
& u(x, 0)=u_{i},
\end{aligned}
$$

where $x_{i}$ is a fixed point in $(0, L)$. The first of these represents an initial aggregation of cells at the posterior marginal zone. We assume zero flux boundary conditions on both the cells and the attractant, and therefore

$$
D_{n} \frac{\partial n}{\partial x}-n \chi_{0} \frac{\partial u}{\partial x}=\frac{\partial u}{\partial x}=0 \quad \text { on } \quad x=0,1,
$$

which stipulates that cells do not leave the area pellucida and enter the area opaca. This is certainly an oversimplification, since at later stages of regression Hensen's node becomes intermixed with cells in the developing tail bud, but at present there is little information on the details of this process.

We first suppose that there is no cell growth, which models a situation in which cell movement takes place on a faster time scale than cell division. This may be a good approximation during progression, which occurs relatively quickly. We therefore take $f_{1}(n, u)=0$ and choose chemical kinetics

$$
f_{2}(n, u)=g(n)-\beta n u-\delta u
$$


(a)

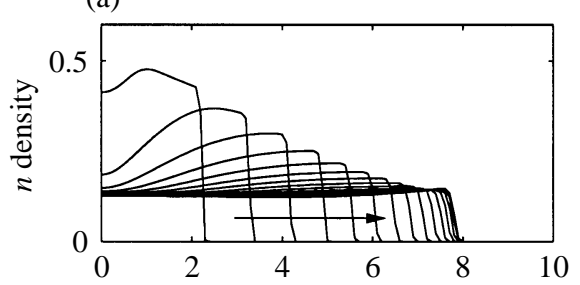

(c)

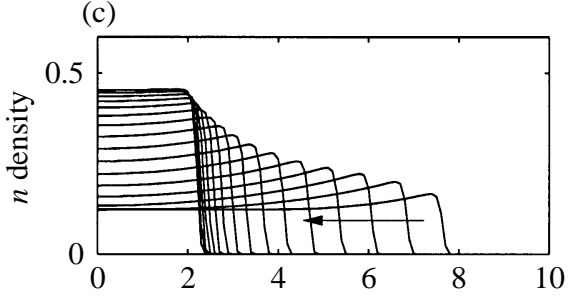

(b)

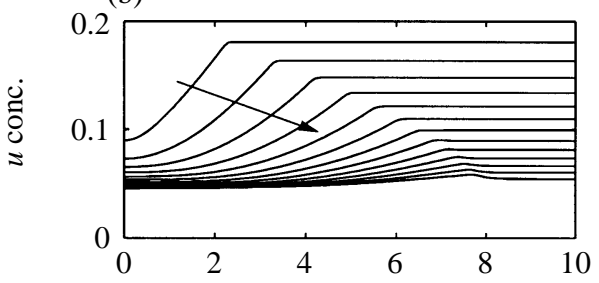

(d)

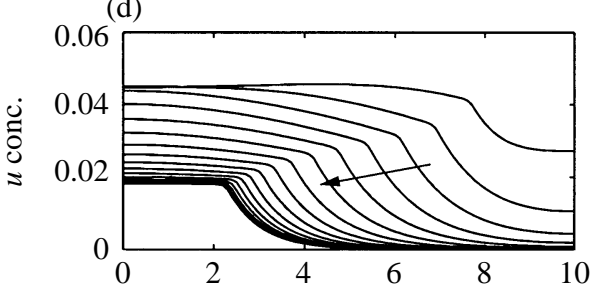

Figure 2. Numerical simulation of the governing evolution equations (1). (a) Forward propagation of the cell density. Each plot represents 10 units of simulation time. (c) Reverse movement of the cell population. The curve of furthest $x$-extension corresponds to $t=200$, and subsequent curves are at increments of 100. (b) and (d) Plots of the chemical concentrations corresponding to the cell densities in (a) and (c). In all panels arrows represent the direction of wave movement. Numerical simulations performed using NAG library routine D03 PGF on the domain $[0,10]$ and parameter values $D_{n}=0.001$, $D_{u}=0.005, \chi_{0}=2.0, \beta=0.1, n_{i}=1.0, \gamma=0.001, \delta=0.01, n_{1}=0.01, x_{i}=1.0$, $u_{i}=0.2$.

in equations (1)-(2). The chemical kinetic terms represent, respectively, chemical production by the cells, chemical degradation by the cells and natural chemical decay, where decay rates $\beta$ and $\delta$ are positive constants. We assume chemical production is switched on above a threshold cell density, $n_{1}$,

$$
g(n)= \begin{cases}\gamma & \text { if } n \geq n_{1} \\ 0 & \text { if } n<n_{1}\end{cases}
$$

The time evolution of numerical solutions to equations (1) and (2) is plotted in Fig. 2. Clearly, the chemotactic model is capable of demonstrating forward and reverse wave movement, with a distinct difference in time scales of the respective movements: in outward movement, there is a steep positive gradient, resulting in fast cell movement. During reverse movement this gradient is shallower. Intuitively, we can explain forward and reverse propagation as follows. Initially, degradation occurs faster nearer the lower boundary due to cells. This creates a chemotactic gradient, resulting in cell movement. Ahead of the cell wavefront, chemical decay terms lower the chemical concentration, which eventually drops to a level lower than that behind the wave. This results in a switch of the chemical gradient. Chemotactic motion pulls the cells backwards, where they reaggregate at the lower boundary. 
The cell density initial conditions have been chosen as an idealization for the aggregation of cells occurring at the posterior region of the embryo prior to streak formation. From a biological standpoint, it is perhaps important to consider whether smoother aggregates can give rise to the same generic movements. Numerical simulations suggest that smoother initial conditions sharpen to demonstrate the same generic movements as shown in Fig. 2. Realistic chemical diffusion coefficients are much larger than cell diffusion coefficients. Numerical simulations demonstrate the same behavior for chemical diffusion coefficients increased by up to two orders in magnitude.

3.0.1. Analysis of forward movement. To facilitate analysis of the model, we non-dimensionalize it by choosing scales such that $\xi=x / L, \tau=\delta t, \hat{n}=n / N_{0}, \hat{u}$ $=u / u_{0}$, and we define

$$
\theta_{1}=\frac{D_{n}}{\delta L^{2}}, \quad \theta_{2}=\frac{\chi_{0}}{\delta u_{0} L^{2}}, \quad \theta_{3}=\frac{D_{u}}{\delta L^{2}} .
$$

This gives the following non-dimensional model:

$$
\begin{aligned}
& \frac{\partial \hat{n}}{\partial \tau}=\theta_{1} \frac{\partial^{2} \hat{n}}{\partial \xi^{2}}-\theta_{2} \frac{\partial}{\partial \xi}\left(\hat{n} \frac{\partial \hat{u}}{\partial \xi}\right), \\
& \frac{\partial \hat{u}}{\partial \tau}=\theta_{3} \frac{\partial^{2} \hat{u}}{\partial \xi^{2}}+\hat{g}(\hat{n})-\hat{\beta} \hat{n} \hat{u}-\hat{u}
\end{aligned}
$$

where $\hat{\beta}$ and $\hat{g}(\hat{n})$ are appropriately scaled according to the non-dimensionalization. For the parameters of Fig. 2, $\theta_{1}=10^{-3}, \theta_{2}=2.0$, and $\theta_{3}=5 \times 10^{-3}$. We choose $u_{0}=N_{0}=1$, and if we assume that $\partial^{2} \hat{n} / \partial \xi^{2}$ is not too large, then the dominant part of equation (8) is given by

$$
\frac{\partial \hat{n}}{\partial \tau} \sim-\theta_{2} \frac{\partial}{\partial \xi}\left(\hat{n} \frac{\partial \hat{u}}{\partial \xi}\right)=-\theta_{2}\left[\frac{\partial \hat{n}}{\partial \xi} \frac{\partial \hat{u}}{\partial \xi}+\hat{n} \frac{\partial^{2} \hat{u}}{\partial \xi^{2}}\right] .
$$

Under the further assumption that either $\partial^{2} \hat{u} / \partial \xi^{2}$ or $\hat{n}$ is small, the above equation is effectively hyperbolic with speed

$$
\theta_{2} \frac{\partial \hat{u}}{\partial \xi} .
$$

This quantity is not constant in time or space, and it shows that the wave reverses whenever $\partial \hat{u} / \partial \xi$ changes sign. None the less, this wave speed provides a valid approximation during progression, as is shown in Fig. 3, where this value is plotted against the numerically-determined wave speed.

We can also obtain an approximation for the time of forward propagation as follows. If we assume that concentration gradients are negligible ahead of the 

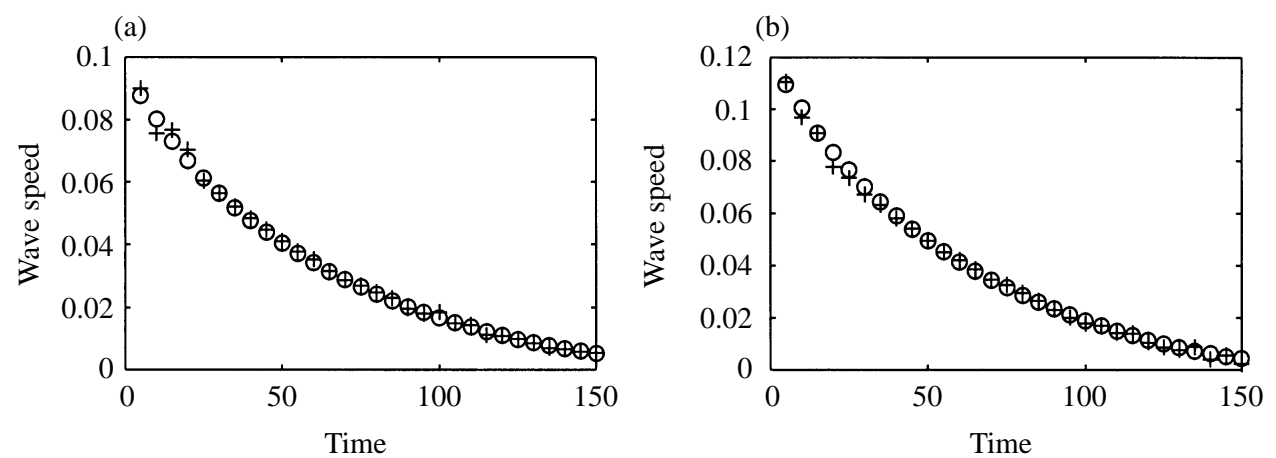

Figure 3. Comparison between the numerically-computed wave speed (cross) and expression (11) (circle). We calculate the wave speed numerically by tracking the point for which $n=0.05$. Since the attractant gradient is a function of time, we use the maximum value of $\hat{u}_{\xi}$ at each time instant in computing the speed. In panel (a) $\chi_{0}=1.0$, and in panel (b) $\chi_{0}=1.5$. Other parameters are as in Fig. 2 .

wavefront (as measured by the threshold density $n_{1}$ ), we find that since $g(n)=0$ ahead of the wave

$$
\hat{u}^{+}(\tau)=u_{i} \exp (-\tau)
$$

where $\hat{u}^{+}$is the attractant concentration ahead of the wave. Sufficiently far behind the wavefront we can neglect gradients of the attractant and assume that the reaction terms are in quasi-equilibrium. As a result, behind the wavefront we can write

$$
\hat{u}^{-}(\tau)=\frac{\hat{\gamma}}{\hat{\beta} \hat{n}^{-}(\tau)+1},
$$

where $\hat{u}^{-}(\tau)$ and $\hat{n}^{-}(\tau)$ are the attractant concentration and cell density, respectively, far behind the wave. The chemical gradient at the threshold density switches at a time $\tau^{*}$ such that $\hat{u}^{+}\left(\tau^{*}\right)=\hat{u}^{-}\left(\tau^{*}\right)$. Reverting to the dimensional scales we find that the time is given by

$$
T=\frac{1}{\delta} \ln \left\{\frac{u_{i}\left(\beta n^{-}+\delta\right)}{\gamma}\right\}
$$

This provides an approximation for the time of forward movement if we know the cell density behind the wave. For the parameters used in Fig. 2 and the computed cell density behind the wave, which is 0.166 , this estimate for the time of forward propagation is $T=167$. In light of approximations necessary to obtain this, it compares very favorably with the time of $T=155$ obtained from the simulation. The analytical expression predicts that increasing the decay rate $\delta$ or the production rate $\gamma$ of the attractant leads to a decrease in the period of forward propagation, whereas an increase in the density-dependent decay rate $\beta$ of the attractant or of the initial density $u_{i}$ leads to an increase in the period of forward propagation. We 

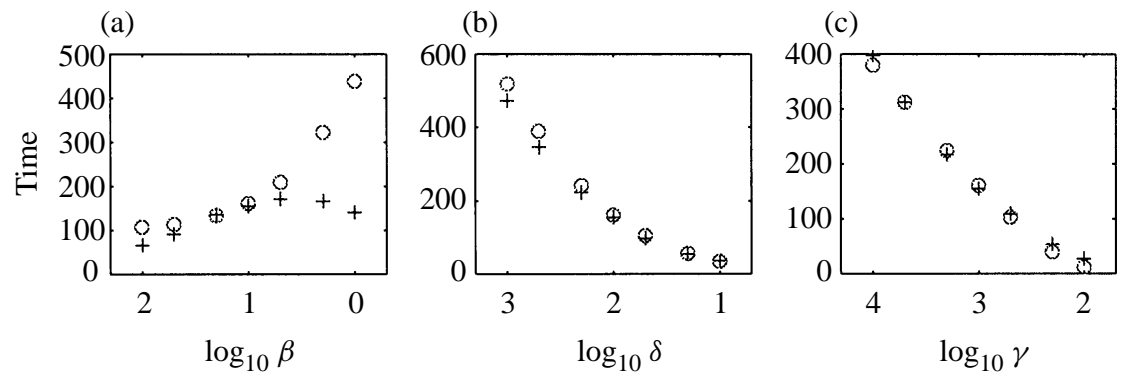

Figure 4. A comparison of the estimated reversal times (circles) and the numericallycomputed times (crosses) for variations of the parameters $\beta,(a), \delta$, (b), and $\gamma$ (c).

Table 1. Analytical upper and lower bounds and numerical determination of the time of forward propagation in the model, equations (1)-(2). 'Normal' refers to parameters used in Fig. 1. Subsequent predictions made for the change of parameter indicated.

\begin{tabular}{cccc}
\hline Parameter change & Lower bound & Upper bound & Numerical \\
\hline Normal & 69 & 309 & 155 \\
$\beta \rightarrow \beta / 2$ & 69 & 248 & 95 \\
$\gamma \rightarrow \gamma / 2$ & 139 & 378 & 200 \\
$u_{i} \rightarrow u_{i} / 2$ & 0 & 240 & 96 \\
\hline
\end{tabular}

have calculated this estimate for variations of these parameters and compared the predicted time of reversal with that obtained from a solution of the full equations. The estimate is remarkably accurate for variations over orders of magnitude in $\delta$ and $\gamma$, but only accurate for small $\beta$, as is shown in Fig. 4. At larger $\beta$, decay of the chemical in the presence of cells is fast, resulting in a sharp concentration gradient. The result is a residue of cells trapped at the boundary which are unable to detect the gradient ahead of them. Thus, profiles do not satisfy the assumptions made in deriving the approximation. Furthermore, since $n_{i}>n^{-}>0$, we are able to obtain upper and lower bounds for this time. In Table 1 we compare these analytical bounds with the numerically evaluated times for a variety of parameter changes.

Forward movement takes place only when a positive chemical gradient develops initially. This is interpreted by requiring the initial rate of chemical decay ahead of the wave to be lower than that behind the wave. Since ahead and behind the wave we have, respectively,

$$
\frac{\partial u^{+}}{\partial t}=-\delta u \quad \text { and } \quad \frac{\partial u^{-}}{\partial t}=\gamma-\beta n u-\delta u,
$$

substitution of the initial conditions gives the following condition for forward propagation:

$$
\gamma<\beta n_{i} u_{i}
$$


This condition shows that an initial attractant concentration greater than the value at the homogeneous steady state is required for forward movement of the cells.

The non-dimensionalization further indicates the respective speeds of forward and reverse propagation. The time taken for forward propagation is $O(1)$ on the non-dimensional scale. Similarly, as indicated by the speed of reverse movement, equation (11), the time taken for reverse propagation is also $O(1)$. Conversion to the dimensional scales yields, respectively, $O(100)$ and $O(1000)$. Both these times are consistent with the numerics.

3.1. Inclusion of growth and division. We now consider whether similar movements can occur when cell growth and division are taken into account. A model of this type is more likely to be relevant for the modeling of developmental processes, where there is often considerable cell division. For example, prior to streak formation, a high area of mitosis exists at the posterior marginal zone (Spratt, 1966). We choose cell kinetics of the form,

$$
f_{1}(n, c)=r h(u) n\left(1-n / n_{0}\right)-\delta_{n} n,
$$

wherein the first term represents cell mitosis and the second represent cell loss. It is assumed that chemical regulation of cell mitosis takes the step function form ${ }^{\dagger}$ :

$$
h(u)= \begin{cases}1 & \text { if } u \geq u_{1} \\ 0 & \text { if } u<u_{1}\end{cases}
$$

We take the same chemical kinetics as for the zero cell growth model, equation (6), but under the further simplification $\beta=0$ (qualitatively, the same behavior is observed with $\beta \neq 0$ ). We can also set $\delta_{n}=0$ and in this case, a large aggregation of cells builds up at the wave front during reversal. We again assume zero flux boundary conditions and initial conditions as given by equations (3)(4). The steady states for the above system are given by $(0,0)$ and $\left(n^{*}, u^{*}\right)=$ $\left(n_{0}\left(1-\delta_{n} / r\right), \gamma / \delta_{u}\right)$, the latter provided that $\gamma / \delta_{u}>u_{1}$ and $n_{0}\left(1-\delta_{n} / r\right)>n_{1}$. For the remainder of this section we suppose both the steady states exist and set $n_{0}=1.0$.

The solutions obtained by numerical solution of the equations are shown in Fig. 5, where one sees that inclusion of cell growth and division still leads to progression and regression. However, now forward propagation occurs against the chemotactic gradient, i.e., the mechanism that drives forward propagation has

\footnotetext{
$\dagger$ In the context of the present model it is necessary to postulate that cell division depends on the concentration of the chemotactic substance, for without this the resulting equation is simply a Fisher-type equation and this allows only uni-directional propagation. Certainly the reality is more complicated than this, but a similar conclusion could be reached from a model that involves more species and that more naturally entails bi-directional propagation, but our purpose here is simply to demonstrate the persistence of bi-directional propagation in the presence of mitosis
} 
(a)

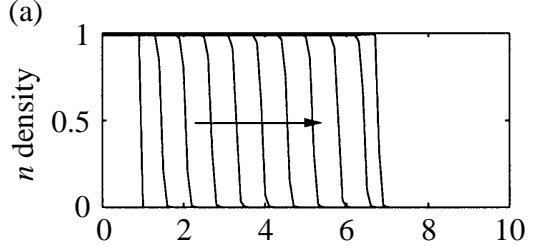

(c)

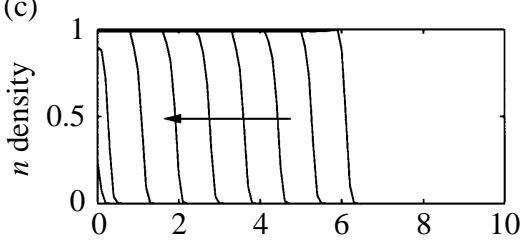

(b)

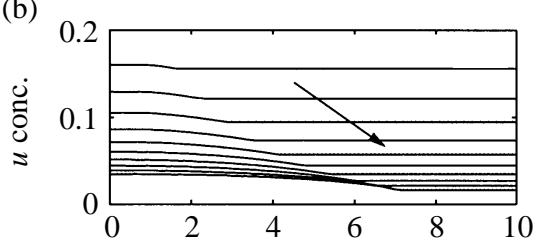

(d)

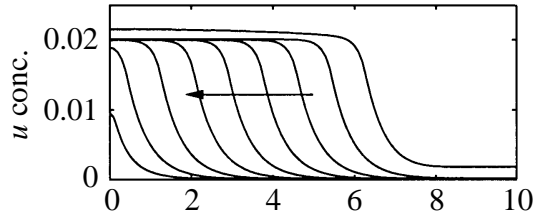

Figure 5. (a) Forward propagation of cells, at time intervals of 5. (b) Corresponding plot for chemical concentrations. (c) Reverse propagation of cells, first plot $t=50$, and subsequent plots at intervals of 50. (d) Corresponding chemical concentrations. Wave direction is indicated by the arrows. $D_{n}=0.001, D_{u}=0.005, \chi_{0}=2.0, r=4.0$, $n_{0}=1.0, \delta_{n}=0.05, u_{1}=0.0195, \delta_{u}=0.05, \gamma=0.001, \beta=0, n_{1}=0.01, n_{i}=1.0$, $x_{i}=1.0, u_{i}=0.2$.

changed, the attractant concentrations are everywhere above the threshold $u_{1}$ during forward propagation. In later stages, cell division is switched off and cells are dragged back along the domain.

The reaction-diffusion equation that describes the evolution of the cell density is a variant on the Fisher equation (Fisher, 1937), with the additional incorporation of chemotactic motion. Subsequently, wave propagation occurs and cells spread outwards across the domain. Due to the changing chemoattractant gradient, however, the wave speed changes during the forward movement. During reverse movement, however, evolution to a traveling-wave-type motion occurs.

3.1.1. Analysis of propagation in the presence of growth. We consider wave motion in a reaction-diffusion model of the form,

$$
\frac{\partial w}{\partial t}=D_{w} \frac{\partial^{2} w}{\partial x^{2}}+f(w)
$$

where $f(w)$ is a 'cubic type' function with two stable steady-state solutions, $w_{1}$ and $w_{3}$. Conversion to the coordinates $z=x-c t$ and assuming traveling wave solutions exist such that $w(x, t)=W(z)$, gives the ODE,

$$
0=D_{w} W_{z z}+c W_{z}+f(W) .
$$

We set boundary conditions, $W(\infty)=w_{1}, W(-\infty)=w_{3}$ and $W_{z}(\infty)=W_{z}(-\infty)$ $=0$. Multiplication by $W_{z}$ and integrating with respect to $z$ gives,

$$
0=\frac{D_{w}}{2}\left[W_{z}\right]_{-\infty}^{\infty}+c \int_{-\infty}^{\infty} W_{z}^{2} d z+\int_{1}^{0} f(W) d W=0 .
$$


Under application of the boundary conditions, rearrangement yields

$$
c=\frac{\int_{0}^{1} f(W) d W}{\int_{-\infty}^{\infty} W_{z}^{2} d z} .
$$

The denominator is always positive, and so the sign of the wave speed is determined by the sign of the integral. More explicitly,

$$
c>0 \text { if } \int_{0}^{1} f(W) d W>0 \quad \text { and } \quad c<0 \text { if } \int_{0}^{1} f(W) d W<0 .
$$

In our model, both fixed points determined through a traveling wave analysis of the system can be shown to be hyperbolic. Consequently, we are unable to apply traditional techniques (Fisher, 1937) to determine a prediction for the minimum wave speed. However, by adopting an analysis similar to the simple system above, we demonstrate how an intuitive understanding for when reverse propagation occurs can be derived. We consider equation (2) describing chemical evolution with kinetics as in equation (6) and $\beta$ set to zero. Transforming to the traveling wave coordinates, $y=x-a t$, and assuming solutions $u(x, t)=U(y)$ and $n(x, t)=N(y)$ gives the ODE,

$$
D_{u} \frac{d^{2} U}{d y^{2}}+a \frac{d U}{d y}+g(N)-\delta_{u} U=0 .
$$

We multiply equation (23) by $\frac{d U}{d y}$ and integrate with respect to $y$ between $-\infty$ and $\infty$. Under the limits, $\frac{d U}{d y}(-\infty)=\frac{d U}{d y}(\infty)=0$, this gives:

$$
a \int_{-\infty}^{\infty}\left(\frac{d U}{d y}\right)^{2} d y+\int_{-\infty}^{\infty}\left(g(N)-\delta_{u} U\right) \frac{d U}{d y} d y=0
$$

Since $\int_{-\infty}^{\infty}\left(\frac{d U}{d y}\right)^{2} d y>0$ and $U(-\infty)=u^{*}, U(\infty)=0$, we have $a<0$ (reverse moving waves) whenever

$$
\int_{0}^{u^{*}}\left(g(N)-\delta_{u} U\right) d U<0 .
$$

$g(n)$ is defined by a simple step function. We define $U_{\text {crit }}$ to be the value of $U$ at the point $y_{1}$ of the traveling wave coordinate system such that $N\left(y_{1}\right)=n_{1}$. The function $g(N)-\delta_{u} U$ is displayed graphically in the left of Fig. 6. This demonstrates that for $U_{\text {crit }}>u^{*} / 2$, the integral, (25), is negative and hence the wave speed is negative. This represents reverse propagation. We plot cell and chemical wave profiles for a specific point in time from the simulations of Fig. 5 in the right of Fig. 6. Validity of the above approach is demonstrated by variation of a model parameter (we choose $c_{1}$ ). As we decrease the value of this parameter the reverse propagating waves switch to forward propagating waves. Calculation of $U_{\text {crit }}$ indicates that the change of wave direction occurs as this value decreases below $u^{*} / 2$, see Table 2. 
(a)

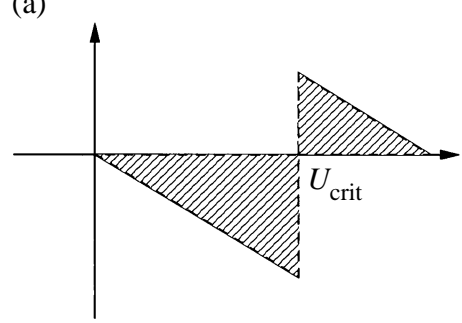

(b)

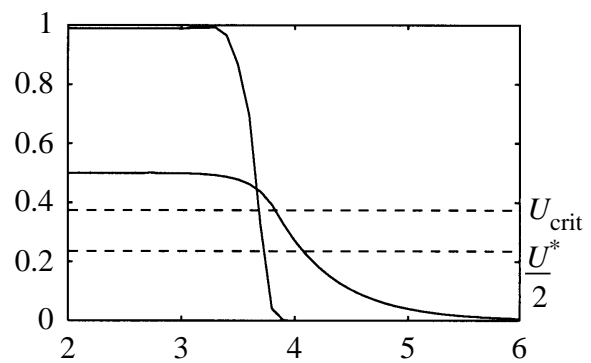

Figure 6. (a) Graphical representation of the function determining sign of the wave speed. The direction of the wave is determined by the size of the shaded area. (b) Traveling wave profiles for cell and chemical profiles. Chemical concentrations have been scaled by 25 for convenience of representation. Profiles taken at $t=250$. Upper dashed line, line shown for concentration of $U_{\text {crit }}$, shown in comparison to $U^{*} / 2$.

Table 2. Table demonstrating the bifurcation from reverse propagating to forward propagating waves during the secondary phase of model evolution as $u_{\text {crit }}$ passes below $u^{*} / 2$.

\begin{tabular}{lccc}
\hline \multicolumn{1}{c}{$c_{1}$} & $u_{\text {crit }}$ & Wave speed & Wave direction \\
\hline 0.0125 & 0.0107 & -0.0021 & Negative \\
0.012 & 0.0103 & -0.0008 & Negative \\
0.01175 & 0.0100 & 0.0 & Zero \\
0.0115 & 0.0098 & 0.0006 & Positive \\
0.011 & 0.0094 & 0.0019 & Positive \\
\hline
\end{tabular}

\section{Movement of The Streak in a Two-dimensional Domain}

The results in one dimension suggest the chemotactic-cell model can replicate movement patterns similar to those shown during progression and regression of the streak. However, if we use the same initial conditions in two dimensions as in one dimension, the desired pattern of cell movement is not obtained. Simulations show that for a uniform initial concentration and an initial cell density representing an aggregation of cells at the posterior marginal zone, cells spread out from the initial mass in a circular manner. Thus some modifications of the model are required to produce movement of the cells towards the center of the domain while maintaining a rod structure.

However there is a simple modification of the initial attractant distribution that produces the desired motion. In the simulation results shown in Fig. 7, the initial conditions are such that the chemoattractant has its highest concentration at the center of the domain (corresponding to the center of the area pellucida) and decreases to zero at the marginal zone. The simulations demonstrate that cells move away from their initial positions towards the center of the domain, forming a rodlike structure during this 'progression'. As cells reach the center of the domain, a bulbous region of highest cell density develops at the most spatially (or ante- 
(a)

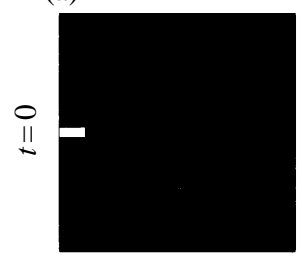

(b)

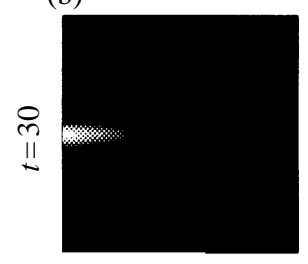

(c)

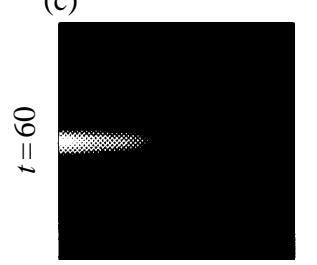

(d)

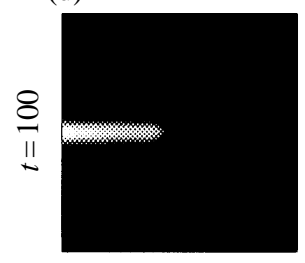

(e)

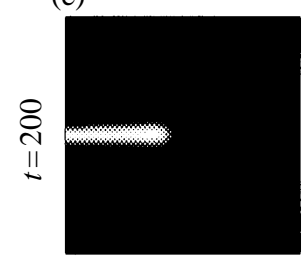

(f)

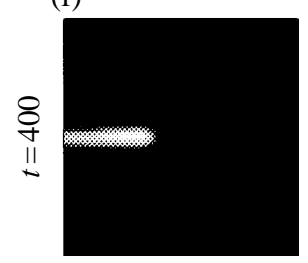

(g)

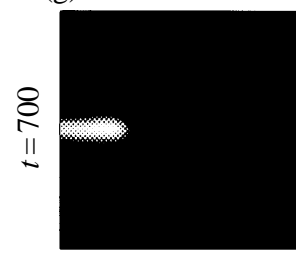

(h)

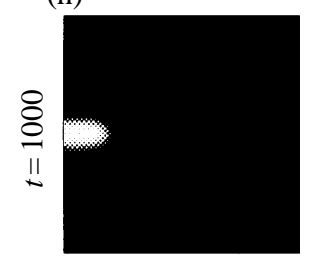

(i)

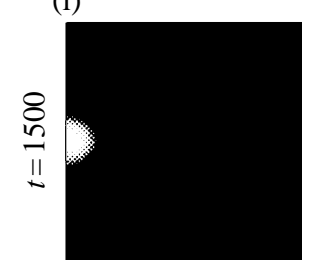

Figure 7. Time sequence showing cell density for the model on a two-dimensional rectangular domain. White represents high cell density, black represents a zero cell density. Simulations demonstrate cell movement across the domain to form a rod which extends approximately half the way across the domain (e). Subsequent development shows a period of reverse movement, which occurs on a slower time scale. Simulations performed with an adapted ADI method with 101 by 101 mesh points. Parameters are $D_{n}=0.001$, $D_{u}=0.01, \chi_{0}=1.0, \beta=0.1, n_{i}=1.0, \gamma=0.001, \delta=0.005, n_{1}=0.01, x_{i}=1.0$, $u_{i}=0.2$, and initial conditions as described in text (maximum initial chemical concentration of 0.2 ).

riorly) extended part of the 'streak'. At this time, progression has ceased, and a period of regression begins with cells moving in a posterior direction, where they reaggregate at the domain boundary. A plausible mechanism for establishing the chemotactic gradient necessary for ingression is as follows. Suppose that all cells in the blastoderm produce the attractant at a constant rate, and that the attractant is degraded by cells in the marginal zone: then the concentration of this species will be highest at the center of the disc, and primitive streak cells can follow the gradient toward the center.

4.1. Comparison with experimental data and model predictions. We have demonstrated above how a simple chemotactic-cell model can accurately convey the principle movements associated with progression and regression of the primitive streak. More specifically, the cells move out to the center of the domain, resulting in a long, thin, rod-like structure reminiscent of the primitive streak. The ac- 
(a)

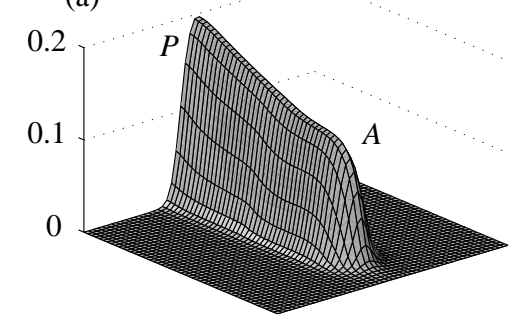

(c)

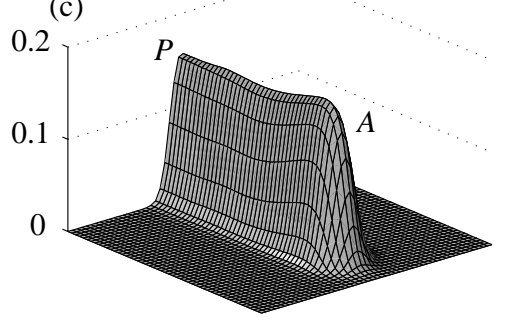

(b)
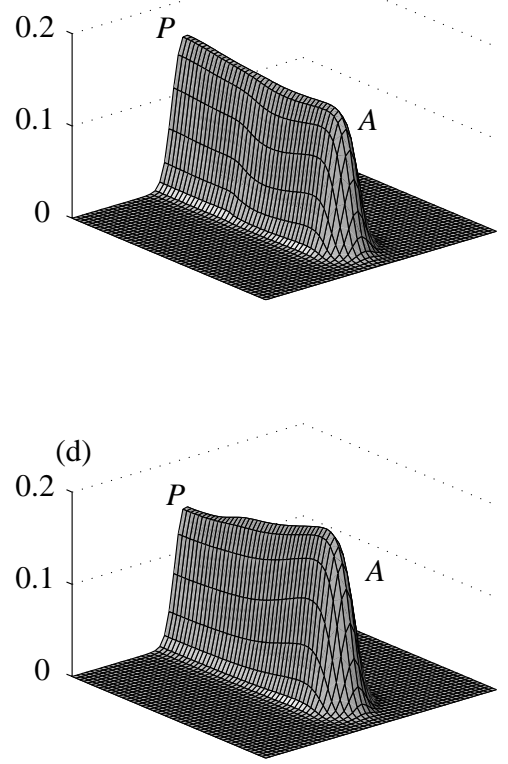

Figure 8. Development of a nodal-like structure at the anterior portion of the developing 'streak'. The highest point of cell density moves from posterior to anterior, creating a bulbous-type structure reminiscent of Hensen's node. This structure is subsequently retained during regression.

tion of chemotaxis results in the majority of cells aggregating at the most anterior part of the 'streak' (see Fig. 8), creating a bulbous-type structure reminiscent of Hensen's node. The cell density patterns during this forward movement have a strong similarity with the results of in situ hybridization experiments showing the spatio-temporal patterning sequence of cells expressing gsc during formation of the primitive streak (Izpisua-Belmonte et al., 1993). The time of progression in our 'streak' is fast with respect to the subsequent movements; cells shoot across the domain as they climb the attractant gradient. This fast movement concurs with observations on progression of the primitive streak on time lapse videos (Vakaet, 1984; Stern, 1990).

During the second stage, cells return to the initial site, reminiscent of the movements during regression of the streak. Labeling of the cells of Hensen's node itself during regression demonstrates that some cells leave the node to form notochord, whereas others remain a part of Hensen's node. We compare the time course of regression charted by Spratt (1947) with the time course in our model in Fig. 6. Clearly, the slopes of the graph show close agreement, with the speed of regression slowing as they approach the boundary.

4.1.1. Ectopic streak development. The ability of specific parts of the embryo to induce an ectopic primitive streak has been identified by a number of experi- 

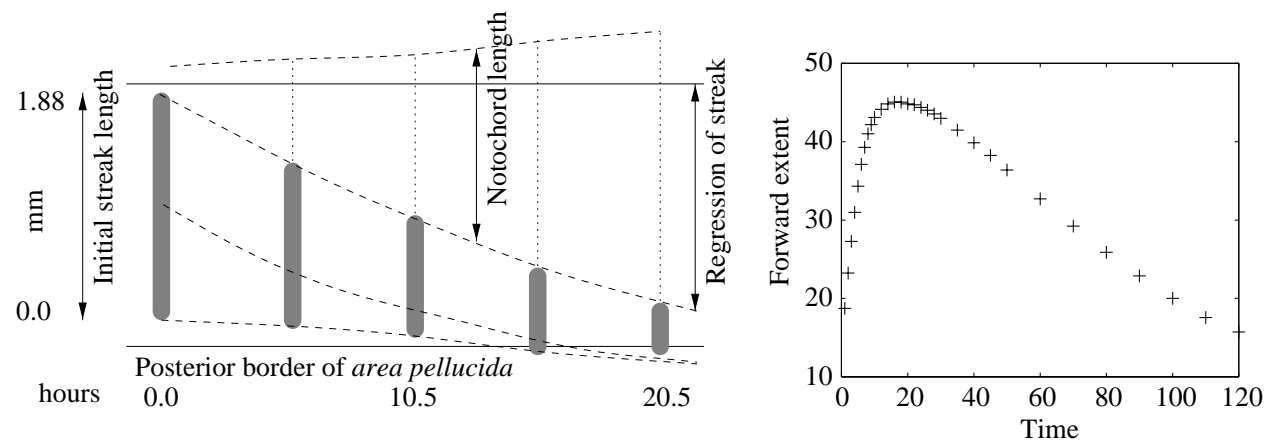

Figure 9. Comparison of experimental data with model data showing spatial extent of primitive streak during regression. Numerical data demonstrate the same slowing in the rate of regression as the streak shortens.

ments. Khaner and Eyal-Giladi (1989) have demonstrated that transplantation of a portion of the posterior marginal zone into the lateral marginal zone of a host embryo induces a second primitive streak to grow at 90 degrees to the host primitive streak (growing from the posterior marginal zone). Similarly, this capability has been demonstrated in grafts of cells of Koller's sickle to lateral portions of a host embryo (Izpisua-Belmonte et al., 1993; Callebaut and Nueten, 1994).

These results are replicated in the model simply by considering initial conditions consisting of two separate cell populations placed at angles representing the posterior marginal zone and lateral marginal zone. The presence of a chemoattractant with maximum concentration at the center of the domain dictates that both populations move across the domain to the center of the area pellucida resulting in ectopic axial structures, see Fig. 10, top. In fact, we predict that progression of a second streak should always take place towards the center of the area pellucida, regardless of the initial position of the transplanted piece, Fig. 10, middle.

The extent of progression is dependent on a variety of factors, for example the initial conditions and model parameters. With respect to development of two ectopic streaks, relatively small variation in factors can result in fusion at the most spatially extended ends, Fig. 10, bottom. Embryos show considerable variation in factors such as size and shape during early development, and we could therefore expect from the model that fusions would occur with a relatively high probability in experiments of the type described above. Indeed, when the region of the posterior marginal zone containing Koller's sickle was grafted to an ectopic site, such fusions were reported in 10 of 37 embryos where a secondary axis was found to develop (Izpisua-Belmonte et al., 1993).

4.1.2. Transplants to central regions and removal of primitive streak cells. We predict that a transplantation to central regions of the area pellucida will not result in the development of a recognizable 'streak', Fig. 11, top. Instead, these cells simply round up to form an aggregation that may itself migrate a little towards the 
Simulation initial conditions

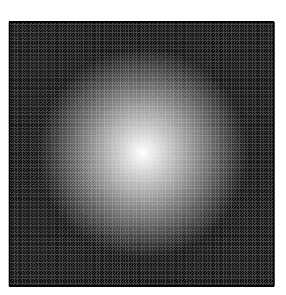

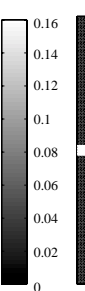

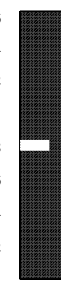

Prediction

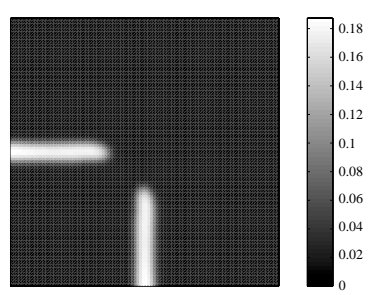

Prediction

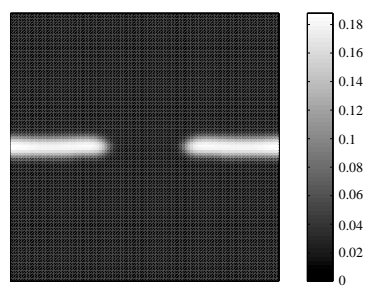

Prediction

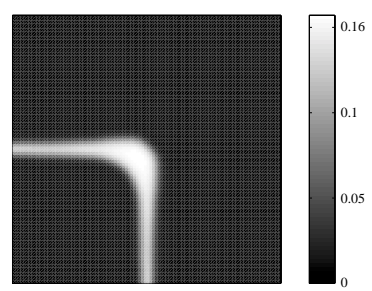

Figure 10. Demonstration of development of ectopic streaks in the two-dimensional model. When a second population of 'able' cells is placed at another point along the marginal zone (top, lateral; middle, anterior), an ectopic streak develops which moves towards the center of the domain. Fairly small changes in model parameters can result in the fusing of these streaks at the anterior ends. In the bottom figures, this has been effected by increasing the concentration gradient of the chemoattractant. 
Simulation initial conditions
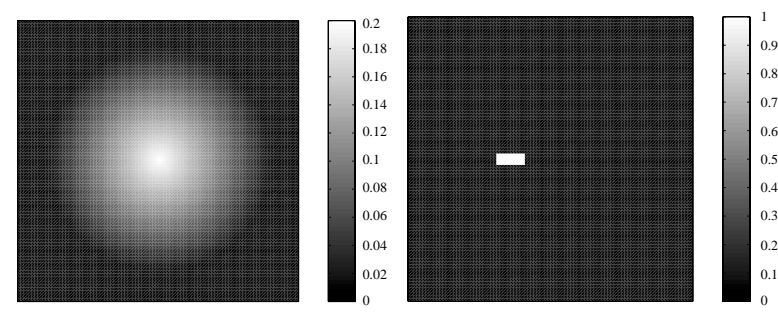

Simulation initial conditions

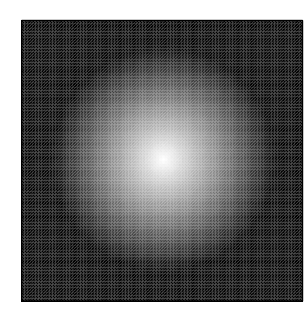

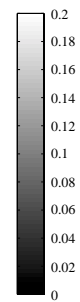

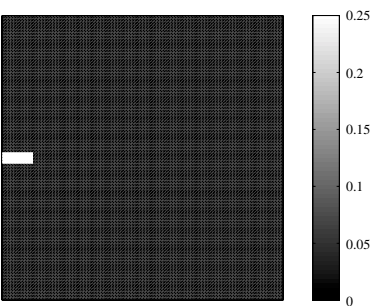

Prediction

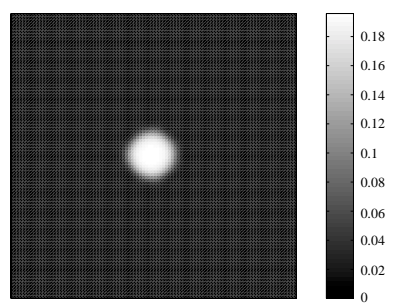

Prediction

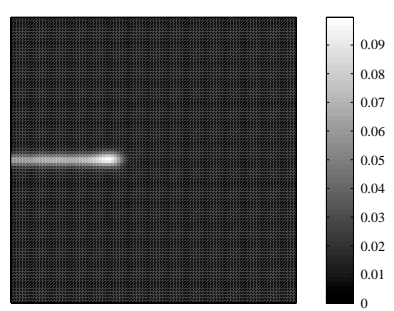

Figure 11. Model predictions of possible experiments. Top, transplantation of the 'able' cells to central portions of the area pelucida does not result in development of a streak of normal morphology. Bottom, removal of a large proportion of able cells still results in development of a streak, albeit of a 'sickly' morphology.

center of the domain.

We can also investigate the effect of the initial cell population on the morphology of the streak that develops. Model simulations suggest that removal of a high proportion of streak marking cells (for example, a reduction of $75 \%$ ) does not significantly effect the extent of streak progression, yet results in a 'sickly' streak, Fig. 11, bottom. These results suggest near normal axial structures can develop despite such severe disturbances.

4.1.3. Blockage of cell movement/attractant gradient experiments. The hypothesis of the center of the domain carrying the information required for correct progression of the streak hints to experimental predictions. For example, a significant portion of the central area pellucida can be removed, and the extent of streak development studied. This experiment is not unrealistic, (Schoenwolf, personal communication)_portions up to one-fifth of the area pellucida can be removed without excessive damage to the embryo. To avoid healing, the space can be filled with beads. These experiments can easily be performed in the model, and we present the results below.

Figure 12(a) demonstrates the result of removing a portion laterally to the central point along the anterior-posterior axis. Subsequent development shows a primitive streak which moves towards the center of the posterior before undergoing slight deviation. When the portion removed is in the anterior half of the area 
(a)

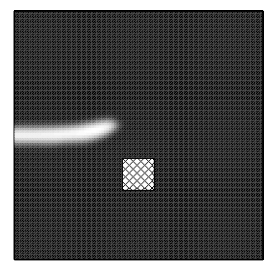

(b)

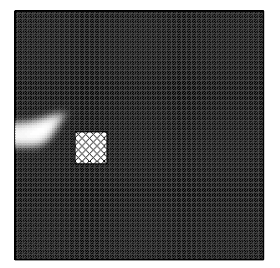

(c)

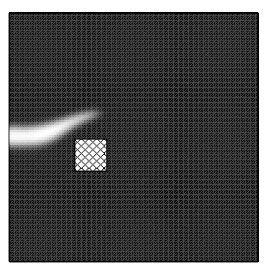

(d)

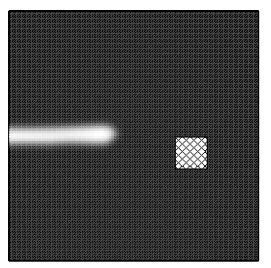

Figure 12. Model predictions for streak development after experimental ablation of certain portions of the area pellucida. See text for details. Ablated portion of area pellucida represented by crossed region. $\mathrm{L}=$ posterior, $\mathrm{R}=$ anterior.

pellucida [Fig. 12(d)], no noticeable effect on primitive streak morphology is observed. Removing portions of the area pellucida at positions along the normal path of the primitive streak during formation impedes normal primitive streak formation [Fig. 12(a) and (b)], yet depending on the position of the removed piece, the primitive streak will sometimes be able to find a path around this removed portion [Fig. 12(c)]. The latter result would not be expected for a guidance mechanism where movement of cells occurs along a pre-determined pathway along the anterior-posterior axis.

\section{Discussion}

In this paper we have demonstrated that chemotactic cell movement coupled with cell-dependent chemical dynamics can produce bi-directional wave propagation in a very simple model. This study has primarily been motivated by the movements taking place during formation and development of the primitive streak in the early stages of chick embryology, but as mentioned earlier, the results are of broader scientific interest. Our analysis of the model leads to a number of realistic and simple experimentally-testable predictions that will help to elucidate the principle mechanisms underlying streak formation.

One of the features of our model here is that it is 'self-contained' in the sense that we have a single mechanism capable of capturing the key features involved in forward and reverse propagation. In many senses this is a naive approach, as it is more likely that not one, but many different mechanisms are responsible. However, in the absence of clear biological evidence favoring a specific mechanism, we were motivated to establish the minimum features a model requires to exhibit the observed behavior, and demonstrate that chemotaxis is a viable mechanism for either one or both of these movements. It is important to realize that a number of other biological processes, for example haptotaxis, contact guidance or adhesion, can give rise to a similar form of mathematical model, and as such are also likely to result in similar predictions. We discuss this further below.

One of the fundamental assumptions of the model developed here is the existence 
of a chemoattractant, with high concentration at the center of the domain, resulting in the outward propagation of the streak. Initial conditions of this form can easily be generated within a biological framework. Two such mechanisms are as follows: firstly, we could consider degradation of the chemical by cells of the area opaca to be stronger, such that the attractant concentration is effectively held at zero within this region. Secondly, we may hypothesis a subpopulation of cells in the center of the domain which produce the attractant prior to streak formation. The former possibility is particularly attractive when one considers the many differences in cell morphology between the two regions. The latter is also not improbable when one considers that it is the cells of the central area pellucida which form the primary hypoblast (Canning and Stern, 1988).

An obvious criticism concerning the two-dimensional results earlier with respect to the cell movements during formation of the streak concerns the extent of forward progression. The model here predicts the development of a primitive streak which extends to approximately the center of the domain, yet common textbook knowledge states that at definitive primitive streak, (stage 4, Hamburger and Hamilton) the streak has extended over approximately two-thirds of the blastoderm. A simple explanation for this anomaly can be provided by considering the blastoderm shape and size changes which occur during progression of the streak. Prior to streak formation, the area pellucida is circular in outline, yet as the streak progresses across the blastoderm, this changes to a pear-like shape, with the narrowing in the posterior half. It is therefore not unreasonable to suppose that the primitive streak extends to a point on the domain which was the approximate center of the blastoderm prior to the shape changes, and that the two-thirds extension is the result of subsequent blastoderm shape changes. Examination of the various stages of primitive streak development provides support for this hypothesis. For example, at stage $3+$, the primitive streak has extended to approximately the center of the still circular AP. Subsequent stages, however, involve transformation to a pear-shaped area pellucida. Early carbon marking experiments performed by Spratt (1946) investigated the movement of cells involved in primitive streak formation. His results indicate that the majority of elongation of the streak at the equivalent of a stage $3+$ blastoderm occurs at the posterior end, and that this growth correlates with the rate of area pellucida extension. Furthermore, cells marked at the anterior end of the streak at this stage (which is at approximately the center of the blastoderm) undergo little forward movement during the latter stages of streak progression: the majority of streak extension occurs in the lengthening posterior portion of the blastoderm. A further test for this hypothesis would be to mark cells across the midline between anterior and posterior sections of the blastoderm prior to streak formation and examine subsequent cell movements.

Early development of the embryo is a very complicated procedure and as yet little is understood of the mechanisms regulating development. Clearly many mechanisms are involved, and as yet we are a long way from a complete model of early development. The model presented here has, for example, ignored questions with 
respect to earlier cell diversity, development of the hypoblast, the potential of the posterior marginal zone to induce axial structures and its inhibition of other regions, the migration of cells to the streak during induction and subsequent development of structures such as the notochord, head process and paraxial mesoderm. It is likely that these processes require many processes which may be highly interconnected. Thus, while demonstrating some generic patterns with respect to primitive streak formation and providing a number of experimental predictions, this model is clearly an over simplification of these stages.

Future improvements could include the extension to a more realistic domain, and the examination of the extent to which cell movements occurring during primitive streak formation account for the general shape changes associated with gastrulation. At the point of egg laying, it is estimated that the blastoderm is composed of approximately 50000-60000 cells (Spratt, 1966). From these numbers, we can estimate the area pellucida is itself composed of approximately 10000 cells. Our current work involves the development of a discrete cell model in which cell movement and other mechanical factors such as cell adhesion are taken into account. In addition to providing a more realistic model of the early embryo where the extensive cell movements are taken into account, this model will allow us to test a number of different mechanisms that have been proposed to account for primitive streak formation such as the convergent-extension idea proposed by Schoenwolf (1991), or whether regression of the primitive streak can be achieved simply by a 'pushing' resulting from cells migrating through Hensen's node to form notochord and other axial structures.

In recent years, investigation of the mechanisms controlling global pattern formation has been replaced with the search of explanations for local development, with the hope that the global aspects will accordingly be revealed (Cooke, 1998b). The expression of c-hairyl, the avian homolog of the Drosophila segmentation gene hairy, however, now forces a new search for the mechanism controlling global pattern formation. The expression indicates a remarkable spatio-temporal sequence and implicates a clear connection with marking the sites of prospective somites. Briefly, as the primitive streak regresses, cells migrate through it to lay down the presomitic mesoderm (PSM), composed of two strips of tissue lying either side of the notochord. Somite pairs form from the PSM, subsequent pairs forming at regular intervals of approximately 90 minutes in an anterior-posterior direction. Expression of $c$-hairyl in cells of the PSM occurs in a cyclic fashion, with a period of 90 minutes. The resultant global behavior shows an initially broad wave of gene expression at the posterior end of the PSM, which narrows as it moves anteriorly to the thickness of a half somite at the anterior end of the PSM. This band of expression remains, and marks the posterior boundary of the presumptive somite. A particularly significant aspect concerning this expression is the widespread, global cell signaling that it indicates and raises the question of how these features are initiated (Cooke, 1998b). The wave of expression initiates from a posterior direction, and thus indicates that the primitive streak may not only have a role in gastrulation 
and the setting of the embryonic axis, but also a role in organizing subsequent development. For example, the act of migration through the primitive streak may act in a manner to reset the internal cellular clock driving the oscillations.

\section{ACKNOWLEDGEMENTS}

K. J. Painter was supported by an EPSRC earmarked studentship in Mathematical Biology and by grant GM29123 from the National Institute of Health. G. Othmer was supported in part by grant GM29123 from the National Institute of Health.

\section{REFERENCES}

Alt, W. (1980). Biased random walk models for chemotaxis and related diffusion approximations. J. Math. Biol. 9, 147-177.

Alt, W., A. Deutsch and G. Dunn (1997). in Dynamics of Cell and Tissue Motion, Birkhäuser.

Armitage, J. P. and J. M. Lackie (1990). in Biology of the Chemotactic Response, Cambridge: Cambridge University Press.

Callebaut, M. and E. V. Nueten (1994). Rauber's (Koller's) sickle: the early gastrulation organizer of the avian blastoderm. Eur. J. Morph. 32, 35-48.

Canning, D. R. and C. D. Stern (1988). Changes in the expression of the carbohydrate epitope HNK-1 associated with mesoderm induction in the chick embryo. Development 104, 643-655.

Cooke, J. (1998a). A gene that resuscitates a theory-somitogenesis and a molecular oscillator. Trends Gen. (Personal edition) 14, 85-88.

Cooke, J. (1998b). A gene that resuscitates a theory-somitogenesis and a molecular oscillator. Trends. Gen. 14, 85-88.

Eyal-Giladi, H. and S. Kochav (1976). From cleavage to primitive streak formation: a complementary normal table and a new look at the first stages of the development of the chick. I General morphology. Dev. Biol. 49, 321-337.

Fisher, R. A. (1937). The wave of advance of advantageous genes. Ann. Eugenics 7, 353369.

Gilbert, S. (1991). Developmental Biology, 3rd edn, Sunderland, MA: Sinauer Assoc., Inc.

Hatada, Y. and C. D. Stern (1994). A fate map of the epiblast of the early chick embryo. Development 120, 2879-2889.

Hume, C. R. and J. Dodd (1993). Cwnt-8C: a novel Wnt gene with a potential role in primitive streak formation and hindbrain organization. Development 119, 1147-1160.

Izpisua-Belmonte, J. E., E. D. Robertis, K. Storey and C. D. Stern (1993). The homeobox gene goosecoid and the origin of organizer cells in the early chick blastoderm. Cell 74, 645-659.

Keller, E. F. and L. A. Segel (1970). Initiation of slime mold aggregation viewed as an instability. J. Theor. Biol. 26, 399-415. 
Keller, R., J. Shih and P. Wilson (1991). Cell motility, control, and function of convergence and extension during gastrulation in Xenopus, in Gastrulation: Movements, Patterns, and Molecules, W. Keller, R. Clark and F. Griffen (Eds), New York, USA; London, UK: Plenum Press.

Khaner, O. (1993). Axis determination in the avian embryo. Curr. Topics Dev. Biol. 28, $155-180$.

Khaner, O. (1998). The ability to initiate an axis in the avian blastula is concentrated mainly at a posterior site. Dev. Biol. 194, 257-266.

Khaner, O. and H. Eyal-Giladi (1986). The embryo-forming potency of the posterior marginal zone in stage X through XII of the chick. Dev. Biol. 115, 275-281.

Khaner, O. and H. Eyal-Giladi (1989). The chick's marginal zone and primitive streak formation. I. Coordinative effect of induction and inhibition. Dev. Biol. 134, 206-214.

Lemaire, L. and M. Kessel (1997). Gastrulation and homeobox genes in chick embryos. Mech. Dev. 67, 3-16.

Marom, K., E. Shapira and A. Fainsod (1997). The chicken caudal genes establish an anterior-posterior gradient by partially overlapping temporal and spatial patterns of expression. Mech. Dev. 64, 41-52.

Mitrani, E. and Y. Shimoni (1990). Induction by soluble factors of organized axial structures in chick epiblasts. Science 247, 1092-1094.

Othmer, H. G. and A. Stevens (1997). Aggregation, blowup and collapse: the ABC's of generalized taxis in reinforced random walks. SIAM J. Appl. Math. 57, 1044-1081.

Patlak, C. S. (1953). Random walk with persistence and external bias. Bull. Math. Biophys. 15, 311-338.

Penner, P. I. and I. Brick (1984). Acetylcholinesterase and polyingression in the epiblast of the primitive streak chick embryo. Roux's Arch. Dev. Biol. 193, 234-241.

Schoenwolf, G. C. (1991). Gastrulation, Chap. Cell movements in the epiblast during gastrulation and neurulation in avian embryos, New York: Plenum Press, pp. 1-27.

Shah, S. B., I. Skromme, C. R. Hume, D. S. Kessler, K. J. Lee, C. D. Stern and J. Dodd (1997). Misexpression of chick Vg1 in the marginal zone induces primitive streak formation. Development 124, 5127-5138.

Spratt, N. T. (1942). Location of organ-specific regions and their relationship to the development of the primitive streak in the early chick blastoderm. J. Exp. Zool. 89, 69-101.

Spratt, N. T. (1946). Formation of the primitive streak in the explanted chick blastoderm marked with carbon particles. J. Exp. Zool. 103, 259-304.

Spratt, N. T. (1947). Regression and shortening of the primitive streak in the explanted chick blastoderm. J. Exp. Zool. 104, 69-100.

Spratt, N. T. (1966). Some problems and principles of development. Am. Zool. 6, 215-254.

Stern, C. D. (1990). The marginal zone and its contribution to the hypoblast and primitive streak of the chick embryo. Development 109, 667-682.

Stern, C. D. (1991). Gastrulation: Movements, Patterns, and Molecules, Chap. Mesodorm formation in the chick embryo revisited, New York: Plenum, pp. 29-41.

Stern, C. D. and D. R. Canning (1990). Origin of cells giving rise to mesoderm and endoderm in the chick embryo. Nature 343, 273-275. 
Vakaet, L. (1984). Early development of birds, in Chimeras in Developmental Biology, N. Le Douarin and A. Mclaren (Eds), London: Academic Press.

Ziv, T., Y. Shimoni and E. Mitrani (1992). Activin can generate ectopic axial structures in chick blastoderm explants. Development 115, 689-694.

Received 5 July 1999 and accepted 29 November 1999 